\title{
Effect of Sodium Nitrate on Microwave-assisted Synthesis of Ceria Nanocubes
}

Tamra Fisher, ${ }^{1}$ MeiyuWang, ${ }^{2}$ Yousif Ibrahim, ${ }^{1}$ Benjamin Steffensmeier, ${ }^{1}$ and Chin Li Cheung $*, 1$

1 Department of Chemistry, University of Nebraska-Lincoln, Lincoln, NE, 68588, United States

2 Department of Mechanical and Materials Engineering, University of Nebraska-Lincoln, Lincoln, NE, 68588, United States

* Corresponding author. Email address: ccheung2@unl.edu

\begin{abstract}
Fluorite-structured cerium oxide (ceria; $\mathrm{CeO}_{2-\mathrm{x}}, 0 \leq \mathrm{x}<0.5$ ) possesses unique catalytic activity to promote redox reactions. Among the most common facets of ceria, the $\{100\}$ facets of ceria nanocubes have been found to be more catalytically active as well as better noble metal supports than the $\{111\}$ facets of ceria nanopolyhedra. Here we report a facile microwave-assisted synthesis of ceria nanocubes using sodium nitrate as the surface modifying agent (SMA). Microwave methods directly scaled from conventional hydrothermal parameters were found to produce mixtures of ceria nanocubes and ceria nanorods. The effect of sodium nitrate on the shape-control growth of ceria nanocubes was investigated by using various mole ratios of sodium nitrate to the cerium nitrate precursor in the synthetic study. From our morphological analysis of resulting ceria products, the mechanistic roles of the SMA were postulated to destabilize the growth of $\{110\}$ and $\{111\}$ facets of ceria nanocrystals as well as influencing the incorporation rate of precursors on the surfaces of ceria crystals.
\end{abstract}

Keywords: ceria; nanocubes; nanorods; sodium nitrate; microwave 


\section{Introduction}

The availability of facile synthetic methods to synthesize shape-defined nanostructured materials is essential to allow systematic study of their surface properties and to develop their practical applications. Many conventional hydrothermal synthetic methods for nanostructured simple oxides such as zinc oxide [1, 2], titanium dioxide [3, 4], and cerium oxide $[5,6]$ require conventional heating the precursors in autoclaves for ten or more hours. Hence, the use of microwave heating, which allows uniform and fast heating and cooling, have been intensively explored [7-9]. However, the original synthetic parameters in reported hydrothermal syntheses of nanomaterials cannot be often transposed for syntheses with microwave heating. Especially for materials with large loss tangent, fast microwave heating can alter the kinetics of nucleation, facet growth rate and hence the shapes of nanocrystals [10]. Thus, specific surface modifying agents engineered to bind to targeted facets and control crystal facet growth rate are usually needed for shape-control syntheses using microwave heating.

Among common facets of ceria nanocrystals, the $\{100\}$ facets of ceria nanocubes have been found to be more catalytically active as well as better noble metal supports than the common $\{111\}$ and $\{110\}$ facets of ceria nanopolyhedra [11]. Herein we report our study of a microwave-assisted synthetic method for fluorite-structured cerium oxide (ceria; $\mathrm{CeO}_{2-\mathrm{x}}, 0 \leq \mathrm{x}<0.5$ ) nanocubes using sodium nitrate as the surface modifying agent (SMA). The use of chemical precursors composed of various mole ratios of SMA to cerium nitrate was investigated to elucidate the effect of SMA on the shape-control growth of ceria nanocubes. Our microscopy analysis of the as-synthesized products revealed that the additional sodium nitrate likely functioned by destabilizing the growth of $\{111\}$ and $\{110\}$ facets of ceria nanocrystals.

\section{Material and Methods}

Nanostructured ceria were synthesized by incorporating microwave heating and sodium nitrate in a previous hydrothermal method for ceria nanocubes that employed cerium nitrate and concentrated sodium hydroxide as the chemical precursors $[12,13]$. To systematically study the effect of sodium nitrate on the growth of ceria nanocubes using microwave heating, ceria products were made from reactants with mole ratios of cerium nitrate to sodium nitrate of 1:0, 1:0.03125, 1: 0.125 and 1:2. For example, for the synthesis with cerium nitrate to sodium nitrate of 1 : 0.125, $8 \mathrm{mmol}$ of cerium nitrate, $1 \mathrm{mmol}$ of sodium nitrate, $960 \mathrm{mmol}$ of sodium hydroxide were added to $160 \mathrm{~mL}$ of $18.2 \mathrm{M} \Omega \cdot \mathrm{cm}$ deionized water and stirred for $20 \mathrm{~min}$. in an ice bath, followed by $10 \mathrm{~min}$. at room temperature. The resulting solution was then divided amongst EasyPrep microwave Teflon vessels (CEM Inc, Matthews, NC) in 40 
mL aliquots. The capped vessels were heated in a MARS6 laboratory microwave (CEM Inc, Matthews, NC) to 180 ${ }^{\circ} \mathrm{C}$ in $15 \mathrm{~min}$, held at $180{ }^{\circ} \mathrm{C}$ for $2 \mathrm{~h}$., and then cooled to room temperature over $30 \mathrm{~min}$. The products were centrifuged at 2600 R.P.M. in five $30 \mathrm{~min}$. intervals to remove excess sodium hydroxide. After each centrifugation, the supernatant was removed and the precipitates were re-suspended and centrifuged twice with deionized water and twice with ethanol. The rinsed precipitates were dried overnight in a convection oven at $50{ }^{\circ} \mathrm{C}$. Lastly, the dried products were crushed with a spatula and dried at $50{ }^{\circ} \mathrm{C}$ for an extra hour before storage.

The morphology and atomic structures of the as-synthesized ceria products were investigated using transmission electron microscopy (TEM) and powder X-ray diffraction (XRD). TEM images of samples dispersed on copper TEM grids were recorded with a Hitachi H7500 TEM (Hitachi High-Technologies America, Inc., Pleasanton, CA) operated at $120 \mathrm{kV}$ and a FEI Tecnai Osiris S/TEM (FEI, Hillsboro, OR) operated at $200 \mathrm{kV}$. The XRD patterns were recorded with a PANalytical Empyrean diffractometer using a $\mathrm{Cu} \mathrm{K} \alpha \mathrm{X}$-ray source with an average wavelength of $1.544 \AA$. The ICCD card \# 04-013-4361 was used to index the diffraction peaks corresponding to the Fm-3m structure of $\mathrm{CeO}_{2}$.

\section{Results and Discussions}

The morphology and atomic structures of the as-synthesized ceria were ascertained through XRD and TEM analyses. The XRD patterns showed that all synthesized samples exhibited the same Fm-3m crystal symmetry as that of bulk fluorite-structured $\mathrm{CeO}_{2}$. (Fig. 1) TEM data revealed that, contrary to the desired cube-selective growth under established conventional hydrothermal conditions, scaled heating time parameters for the microwave-assisted synthetic process yielded a mixture of ceria nanorods and ceria nanocubes. However, the addition of minute amount of sodium nitrate as the surface modifying agent (SMA) to the reaction mixture yielded off-white precipitate products. Our TEM analysis revealed a cube-selective ceria

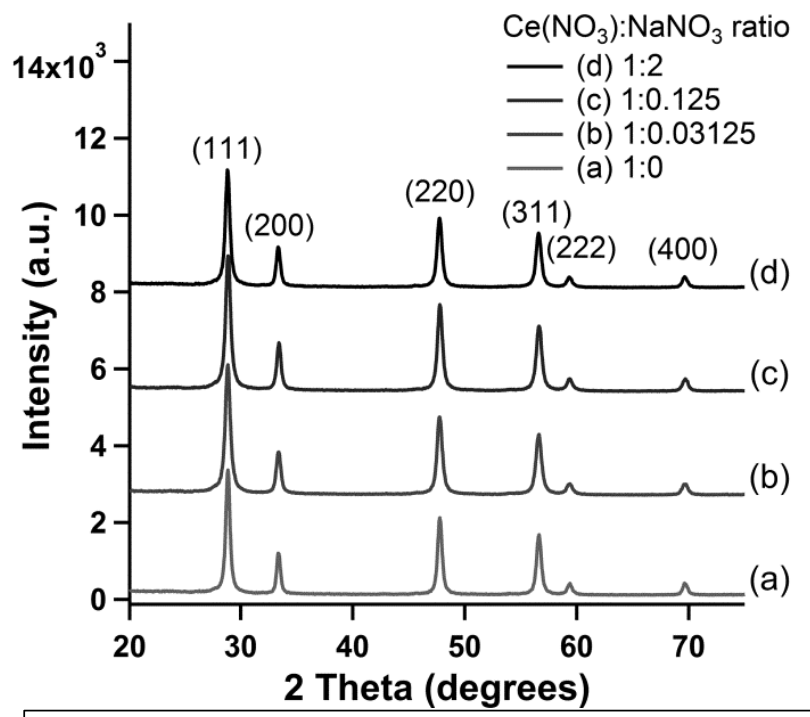

Fig. 1. X-ray powder diffraction patterns of nanostructured ceria samples synthesized from different mole ratios of cerium nitrate and sodium nitrate: (a) 1: 0, (b) 1:0.03125, (c) 1:0.125 and (d) 2:1. 


\section{Increase in $\mathrm{NaNO}_{3}$ concentration}

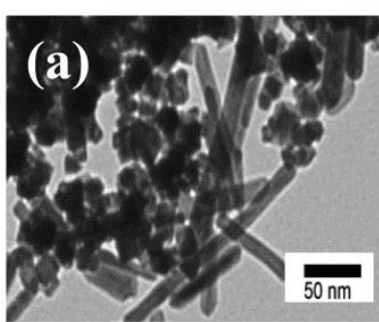

(e)

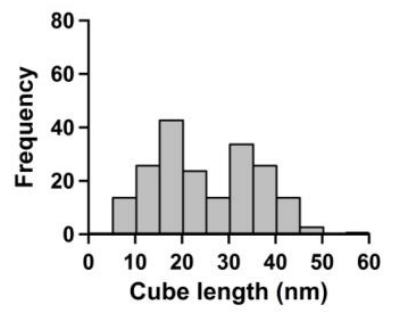

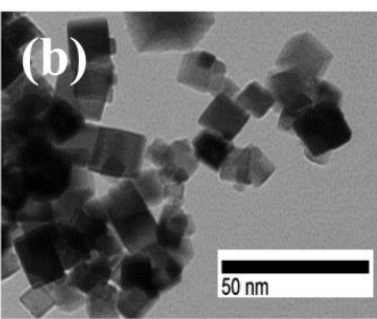

(f)

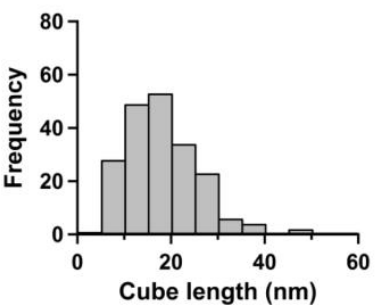

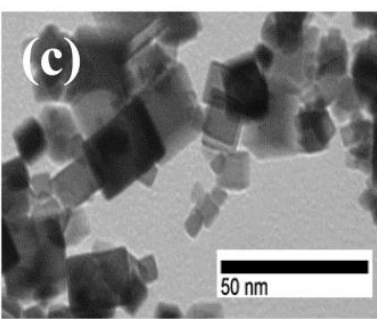

(g)

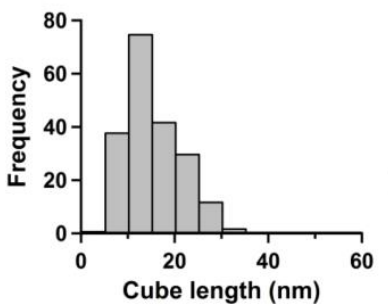

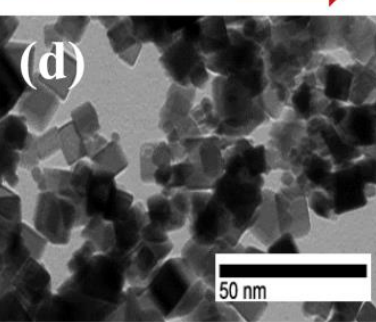

(h)

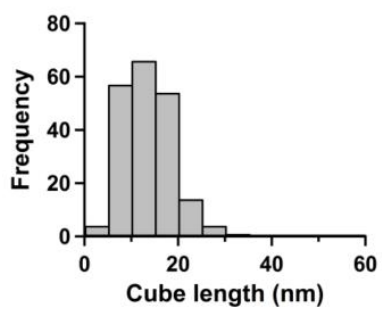

Fig. 2. (a-d) TEM images and (e-h) size distributions of nanostructured ceria samples synthesized from different mole ratios of cerium nitrate and sodium nitrate: (a, e) 1:0, (b, f) 1:0.03125, (c, g) 1:0.125, and (d, h) 1:2.

nanostructure growth using the SMA. (Fig. 2 a-d) Ceria nanocubes made with various mole ratios of added SMA were found to possess slight but noticeable shifts in cube length distributions. (Fig. 2 e-h) As the mole ratio of added SMA increased, the cube length distribution became narrower and more weighted toward smaller values (10$15 \mathrm{~nm})$. Our results illustrated that the addition of sodium nitrate did not only promote the growth of ceria nanocubes but also deter the growth of ceria nanorods in the products. As the SMA concentration increased in the reaction mixtures, few ceria nanoparticles with increasing regularity were also sporadically observed.

High resolution transmission electron microscopy (HRTEM) further revealed the effect of sodium nitrate on the facet growth of ceria crystals. HRTEM image of a ceria nanocube synthesized with

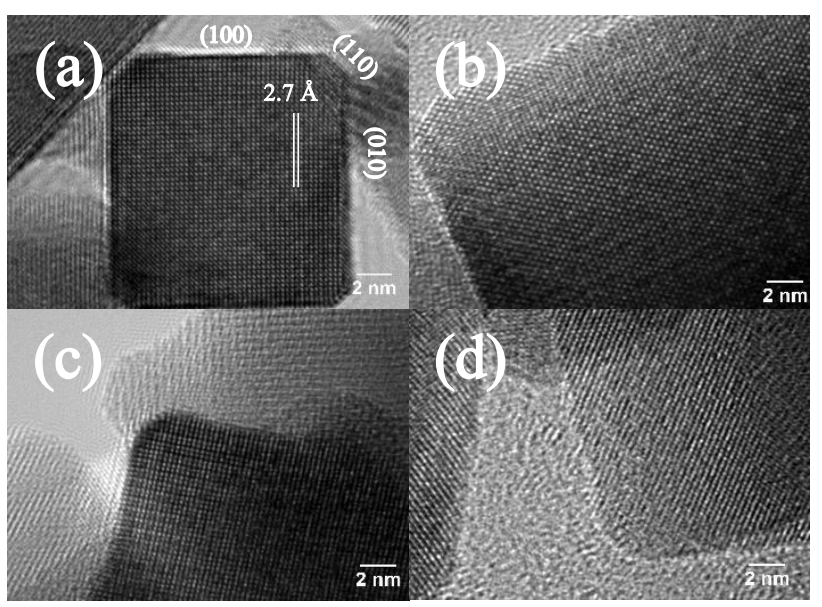

Fig. 3. High-resolution TEM images of nanostructured ceria samples synthesized from different ratios of cerium nitrate precursors and sodium nitrate precursors: (a)-(b) 1:0 and (c)-(d) 1:2. cerium nitrate and no sodium nitrate displayed defined $\{100\}$ surfaces as well as $\{110\}$ at the edges. (Fig. 3a and 
3b) These features are expected of typical nanocube growth from hydrothermal synthesis in previous publications

$[12,14]$. In contrast, though ceria nanocubes synthesized with the SMA addition appeared to display $\{100\}$ surfaces under low magnifications, under HRTEM, the atomic arrangements at the corners and edges of these nanostructures were observed to be rounded and less-defined. For example, upon closer examination of the edges of ceria nanocubes made with 1:2 mole ratio of cerium nitrate and sodium nitrate, the corresponding HRTEM images revealed the rounding of the facets at the corners and edges of the nanocube products. (Fig. $3 \mathrm{c}$ and $3 \mathrm{~d}$ ) These rounded features suggested strong interactions between nitrate and these faceted surfaces and the inhibition of proper $\{111\}$ and $\{110\}$ facet growth. Typical ceria nanorods have either [110] growth direction or [211] growth directions. The ceria nanorods with [110] growth direction have predominantly $\{111\}$ facets exposed whereas those with [211] growth direction possess both exposed $\{100\}$ and $\{111\}$ facets [15]. The absence and prohibited growth of nanorods with the addition of SMA could further infer the facet-specific interactions with excess nitrate.

According to our experimental findings, we propose potential mechanistic roles of sodium nitrate in ceria nanoconstruct growth under microwave-assisted reaction conditions. (Fig. 4) In this proposed mechanism, cerium ions first react with hydroxide ions to form nuclei of cerium hydroxide. Heating to the reactants increases the growth rate of these hydroxide nuclei and assists the dehydration of these hydroxide nuclei into ceria nuclei. The growth of different facets on the ceria nuclei depends on the thermodynamics of the facet formation. When added SMAs bind to

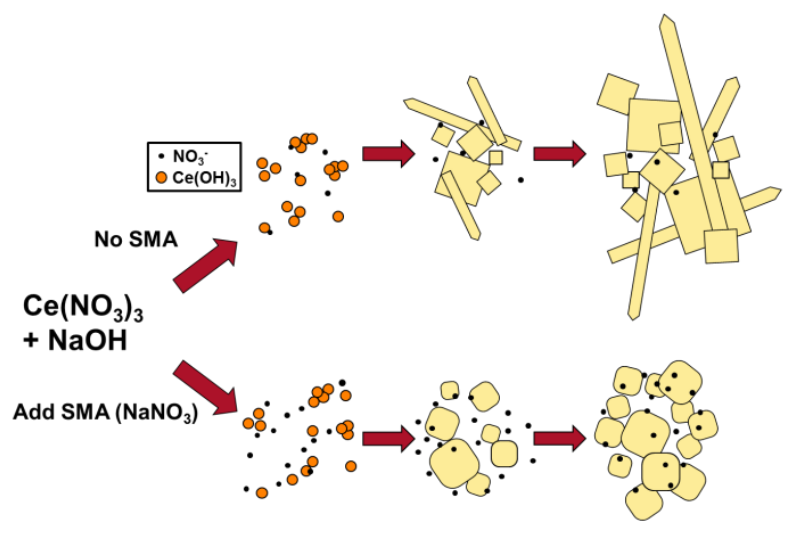
Reaction Time

Fig. 4. Proposed mechanistic roles of the sodium nitrate on the microwave-assisted growth of ceria nanocubes and nanorods. specific facets and modify the facet formation energy, they can either promote or deter the growth of these facets. In the case of synthesis without SMA, owing to the relatively high loss tangent property of ceria [7], the ceria nuclei are postulated to absorb sufficient microwave energy such that they can access and display various facets of low formation energy. Although the cerium nitrate precursor provides nitrate ions which are postulated to stabilize the growth of $\{100\}$ facets of ceria, the concentration of nitrate ions is likely not enough to influence most ceria nuclei because the microwave heating quickly generates a larger density of nuclei than in the case of conventional heating. 
These two factors lead to the growth of different faceted ceria products including nanorods and nanocubes. On the contrary, in the case of synthesis with SMA, the extra nitrate ions increase the chemical potential of these ions to bind more strongly onto the $\{100\}$ facets of ceria nuclei and destabilize the formation of $\{111\}$ and $\{110\}$ facets. Also, more nitrates ions are available to bind onto the ceria nuclei. Consequently, this promotes the growth of mainly ceria nanocubes with rounded edges and corners. As a secondary effect of SMAs, as the ceria nuclei transform into nanocrystals, SMA provides extra nitrate to bind on the facets of ceria nanocrystals and hence decrease the diffusion rate of cerium ions between nanocrystals or the Ostwald ripening effect [16]. This affords more even individual nanocrystal growth rate and thus a narrower length distribution among final nanocube products.

Closer inspection of the HRTEM images in Figures $3 \mathrm{c}$ and $3 \mathrm{~d}$ reveals the presence of small depressions centered on the $\{100\}$ facets of ceria nanocubes grown in an excess of sodium nitrate. The excess nitrate ions likely alter the relative growth kinetics at various locations on the cube facets and lead to differences in precursor concentrations across the facets. The $\{100\}$ facet centers probably have less access to precursors compared to the facets at the edges or corners, leading to the diffusion controlled growth and thus formation of depressions [17]. The diffusion fields are known to be highly symmetric, and tend to favor the growth of symmetric morphologies such as cubes and particles [18]. Hence, in an excess of sodium nitrate, this could also assist the exclusion of nanorod growth while possibly contributing to the formation of nanocubes with a few particles.

\section{Conclusions}

Microwave-assisted synthetic methods were demonstrated to produce cube-selective ceria nanoparticles with the addition of sodium nitrate as the surface modifying agent. The addition of sodium nitrate provided facet-control by destabilizing the growth of the $\{111\}$ and $\{110\}$ facets of ceria nanocrystals as well as influencing the incorporation rate of precursors on the surfaces of ceria crystals. The mechanistic roles of sodium nitrate eliminated the presence of nanorods in the microwave-synthesized products and led to preferred growth of ceria nanocubes with a narrow length distribution.

\section{Acknowledgements}

The authors acknowledge financial support from the National Science Foundation (CHE-1362916). Y.I. and B.S. are grateful for their support from the ACS SEED program and the Nebraska Center for Materials and Nanoscience (NCMN) STEM summer program, respectively. We thank the Morrison Microscopy Center and the NCMN at the 
University of Nebraska-Lincoln and the Central Analytical Facility at the University of Alabama for the use of their facilities.

\section{References}

[1] L.-J. Chen, Y.-J. Chuang, Hydrothermal synthesis and characterization of hexagonal zinc oxide nanorods with a hexamethylenetetramine (HMTA) template-assisted at a low temperature, Materials Letters 68 (2012) 460-462. [2] R. Savu, R. Parra, E. Joanni, B. Jančar, S.A. Eliziário, R.d. Camargo, P.R. Bueno, J.A. Varela, E. Longo, M.A. Zaghete, The effect of cooling rate during hydrothermal synthesis of $\mathrm{ZnO}$ nanorods, Journal of Crystal Growth 311(16) (2009) 4102-4108.

[3] V. Idakiev, Z.Y. Yuan, T. Tabakova, B.L. Su, Titanium oxide nanotubes as supports of nano-sized gold catalysts for low temperature water-gas shift reaction, Applied Catalysis A: General 281(1-2) (2005) 149-155.

[4] T. Peng, D. Zhao, K. Dai, W. Shi, K. Hirao, Synthesis of Titanium Dioxide Nanoparticles with Mesoporous Anatase Wall and High Photocatalytic Activity, The Journal of Physical Chemistry B 109(11) (2005) 4947-4952.

[5] Z. Yang, Y. Yang, H. Liang, L. Liu, Hydrothermal synthesis of monodisperse $\mathrm{CeO}_{2}$ nanocubes, Materials Letters 63(21) (2009) 1774-1777.

[6] Y. Chen, T. Liu, C. Chen, W. Guo, R. Sun, S. Lv, M. Saito, S. Tsukimoto, Z. Wang, Hydrothermal synthesis of ceria hybrid architectures of nano-rods and nano-octahedrons, Materials Letters 96 (2013) 210-213.

[7] F. Bondioli, A.M. Ferrari, C. Leonelli, C. Siligardi, N.A. Hart, N.G. Evans, The application of microwaves in the synthesis of Ce0.9Pr0.1O2 nanostructured powders, Journal of Materials Chemistry 11(10) (2001) 2620-2624.

[8] S. Komarneni, R.K. Rajha, H. Katsuki, Microwave-hydrothermal processing of titanium dioxide, Materials Chemistry and Physics 61(1) (1999) 50-54.

[9] M.L. Dos Santos, R.C. Lima, C.S. Riccardi, R.L. Tranquilin, P.R. Bueno, J.A. Varela, E. Longo, Preparation and characterization of ceria nanospheres by microwave-hydrothermal method, Materials Letters 62(30) (2008) 45094511.

[10] Y. Tao, H. Wang, Y. Xia, G. Zhang, H. Wu, G. Tao, Preparation of shape-controlled $\mathrm{CeO}_{2}$ nanocrystals via microwave-assisted method, Materials Chemistry and Physics 124(1) (2010) 541-546.

[11] K. Zhou, Y. Li, Catalysis Based on Nanocrystals with Well-Defined Facets, Angewandte Chemie International Edition 51(3) (2012) 602-613. 
[12] Y.C. Zhang, M. Lei, K. Huang, C. Liang, Y.J. Wang, S.S. Ding, R. Zhang, D.Y. Fan, H.J. Yang, Y.G. Wang, A facile route to mono-dispersed $\mathrm{CeO}_{2}$ nanocubes and their enhanced photocatalytic properties, Materials Letters 116 (2014) 46-49.

[13] N.K. Renuka, T. Divya, T. Jency Mohan, A facile route to ceria nanocubes, Materials Letters 141 (2015) 107109.

[14] K. Kaneko, K. Inoke, B. Freitag, A.B. Hungria, P.A. Midgley, T.W. Hansen, J. Zhang, S. Ohara, T. Adschiri, Structural and Morphological Characterization of Cerium Oxide Nanocrystals Prepared by Hydrothermal Synthesis, Nano Letters 7(2) (2007) 421-425.

[15] H. Tan, J. Wang, S. Yu, K. Zhou, Support Morphology-Dependent Catalytic Activity of $\mathrm{Pd} / \mathrm{CeO}_{2}$ for Formaldehyde Oxidation, Environmental Science \& Technology 49(14) (2015) 8675-8682.

[16] Z. Yang, Z. K. Zhou, X. Liu, Q. Tian, D. Lu, S. Yang, Single-crystalline ceria nanocubes: size-controlled synthesis, characterization and redox property, Nanotechnology 18(18) (2007) 185606.

[17] C.H.L. Goodman, Crystal Growth: Theory and Techniques Vol. 1: Springer, New York; 1974.

[18] Y. Oaki, H. Imai, Experimental Demonstration for the Morphological Evolution of Crystals Grown in Gel Media, Crystal Growth \& Design 3(5) (2003) 711-716. 

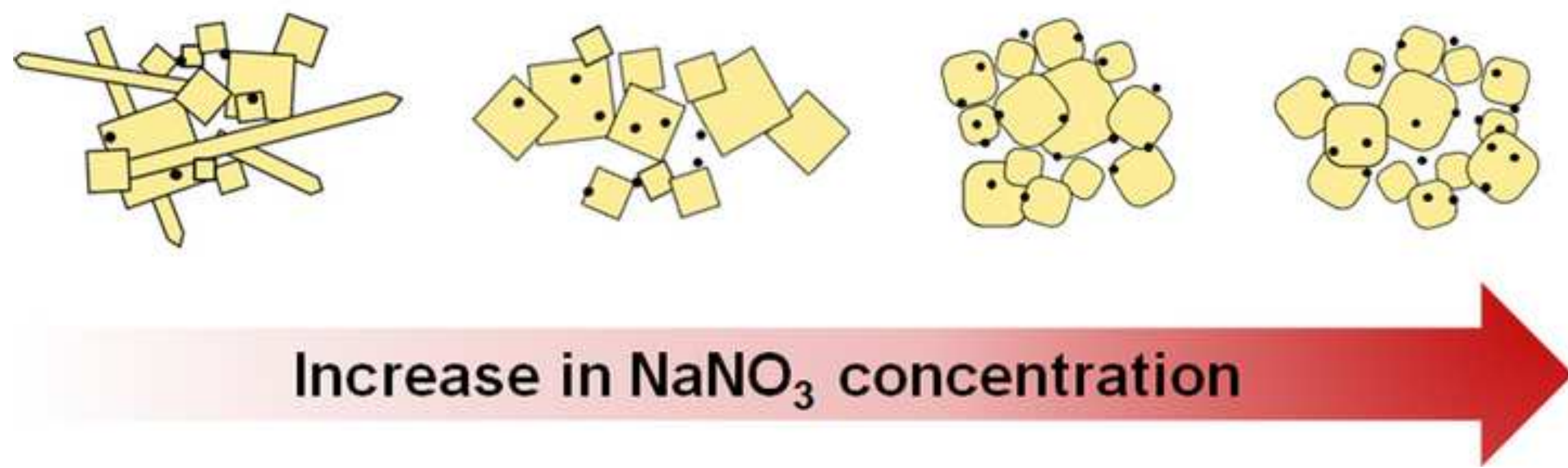

\section{Increase in $\mathrm{NaNO}_{3}$ concentration
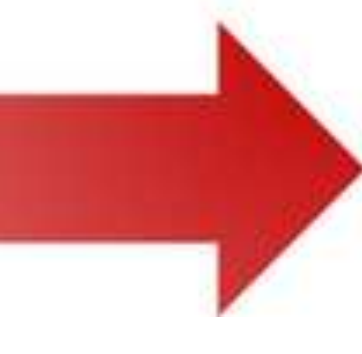

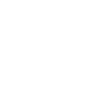

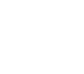

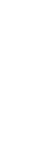

(1)

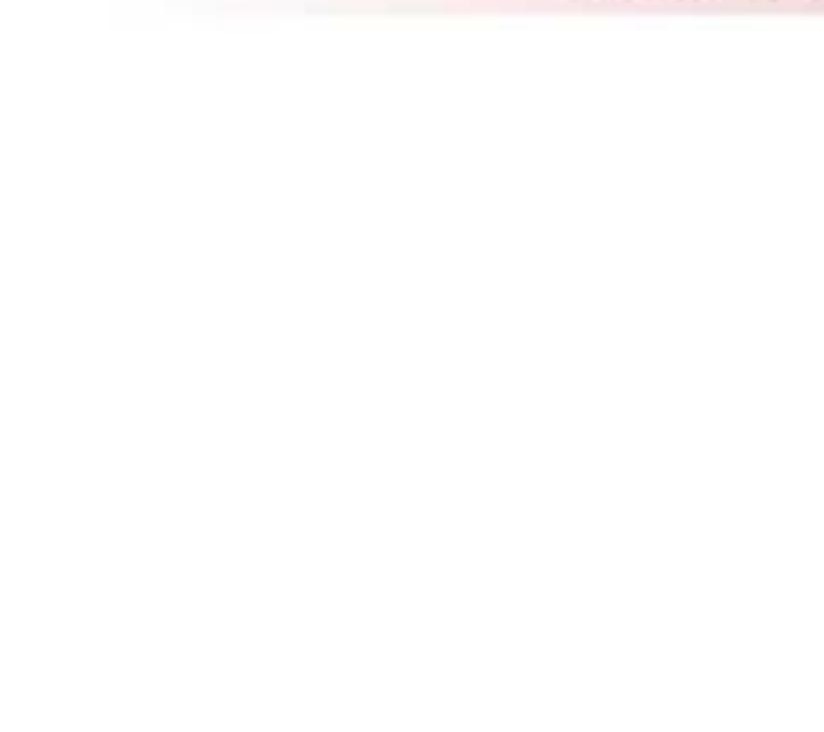

get 